PROCEEDINGS OF THE

AMERICAN MATHEMATICAL SOCIETY

Volume 140, Number 2, February 2012, Pages 575-580

S 0002-9939(2011)11005-0

Article electronically published on June 9, 2011

\title{
THE DISK PROPERTY OF COVERINGS OF 1-CONVEX SURFACES
}

\author{
MIHNEA COLŢOIU AND CEZAR JOIŢA
}

(Communicated by Franc Forstneric)

\begin{abstract}
Let $X$ be a 1-convex surface and $p: \tilde{X} \rightarrow X$ an (unbranched) covering map. We prove that if $\tilde{X}$ does not contain an infinite Nori string of rational curves, then $\tilde{X}$ satisfies the discrete disk property.
\end{abstract}

\section{INTRODUCTION}

Let $X$ be a 1-convex surface, i.e. a two-dimensional complex manifold which is strongly pseudoconvex. Then $X$ is a proper modification of a 2-dimensional normal Stein space at a finite set of points. Let $p: \tilde{X} \rightarrow X$ be an (unbranched) covering map. In this paper we are interested in studying the convexity properties of $\tilde{X}$.

It was remarked in [1] that in general $\tilde{X}$ is not weakly 1-complete (i.e. it might be possible that there is no plurisubharmonic exhaustion function on $\tilde{X})$. This is due to the fact that $\tilde{X}$ might contain an infinite Nori string (necklace), that is, a 1-dimensional connected complex subspace which is non-compact and has infinitely many compact irreducible components. However the main result in 1] shows that $\tilde{X}$ can be exhausted by a sequence of relatively compact strongly pseudoconvex domains with smooth boundary ( $\tilde{X}$ is $p_{3}$-convex in the terminology of [4]). In particular $\tilde{X}$ satisfies the continuous Kontinuitätssatz (the continuous disk property).

In this paper we investigate the discrete disk property for $\tilde{X}$. The main result can be stated as follows (see Theorem 3.4): If $\tilde{X}$ does not contain an infinite Nori string of rational curves, then $\tilde{X}$ satisfies the discrete disk property.

It should be noted that the discrete disk property is a much stronger condition than the continuous one.

\section{Preliminaries}

All complex spaces are assumed of bounded dimension and countable at infinity.

Let $X$ be a complex manifold. We recall that $X$ is said to be 1 -convex if there exists a $\mathcal{C}^{\infty}$ function $\phi: X \rightarrow \mathbb{R}$ such that:

a) $\phi$ is an exhaustion function (i.e. $\{\phi<c\} \Subset X$ for every $c \in \mathbb{R}$ ),

Received by the editors November 23, 2010.

2010 Mathematics Subject Classification. Primary 32F17, 32F10, 32E05, 32E10.

Key words and phrases. Stein spaces, 1-convex spaces, holomorphically convex spaces, discrete disk property.

Both authors were partially supported by CNCSIS Grant PN-II ID_1185, contract 472/2009.

(C)2011 American Mathematical Society 
b) $\phi$ is strongly plurisubharmonic (its Levi form is positive definite) outside a compact subset of $X$.

It is known (see 9]) that this is equivalent to the following condition: there exists a proper surjective holomorphic map $\pi: X \rightarrow Y$ where $Y$ is a normal Stein space with finitely many singular points and there exists a finite subset of $Y, B$, such that $\pi$ induces a biholomorphism from $X \backslash \pi^{-1}(B)$ to $Y \backslash B . \pi^{-1}(B)$ is called the exceptional set of $X$.

A complex space $X$ is called holomorphically convex if for every discrete sequence $\left\{x_{n}\right\}$ in $X$ there exists a holomorphic function $f: X \rightarrow \mathbb{C}$ such that $\lim _{n \rightarrow \infty}|f(x)|=\infty$.

We denote by $\Delta$ the unit disk in $\mathbb{C}, \Delta=\{z \in \mathbb{C}:|z|<1\}$ and for $\epsilon>0$ by $\Delta_{1+\epsilon}$ the disk $\Delta_{1+\epsilon}:=\{z \in \mathbb{C}:|z|<1+\epsilon\}$.

Definition 2.1. Suppose that $X$ is a complex space. We say that $X$ satisfies the discrete disk property if whenever $f_{n}: U \rightarrow X$ is a sequence of holomorphic functions defined on an open neighborhood $U$ of $\bar{\Delta}$ for which there exists an $\epsilon>0$ and a continuous function $\gamma: S^{1}=\{z \in \mathbb{C}:|z|=1\} \rightarrow X$ such that $\Delta_{1+\epsilon} \subset U$, $\bigcup_{n \geq 1} f_{n}\left(\Delta_{1+\epsilon} \backslash \Delta\right)$ is relatively compact in $X$ and $f_{n \mid S^{1}}$ converges uniformly to $\gamma$, we have that $\bigcup_{n \geq 1} f_{n}(\bar{\Delta})$ is relatively compact in $X$.

This definition of the disk property using coronae instead of the boundary $\partial \Delta$ of the unit disk is natural, as one sees by looking at the example $f_{n}: \mathbb{C} \rightarrow \mathbb{C}$, $f_{n}(z)=z^{n}$, which is a sequence that converges to 0 if $|z|<1$ and diverges otherwise.

For $\epsilon>0$ we define $H_{\epsilon} \subset \mathbb{C} \times \mathbb{R}$ as

$$
H_{\epsilon}=\Delta_{1+\epsilon} \times[0,1) \cup\{z \in \mathbb{C}: 1-\epsilon<|z|<1+\epsilon\} \times\{1\} .
$$

Having in mind the definition of the continuity principle (see for example [7, page 47) we introduce the following:

Definition 2.2. A complex space $X$ is said to satisfy the continuous disk property if whenever $\epsilon$ is a positive number and $F: H_{\epsilon} \rightarrow X$ is a continuous function such that, for every $t \in[0,1), F_{t}: \Delta_{1+\epsilon} \rightarrow X, F_{t}(Z)=F(z, t)$ is holomorphic, we have that $F\left(H_{\epsilon_{1}}\right)$ is relatively compact in $X$ for any $0<\epsilon_{1}<\epsilon$.

Clearly the discrete disk property implies the continuous one, but as is shown by the example of Fornaess (see [6]) of an increasing union of Stein open subsets that does not satisfy the discrete disk property, the discrete disk property is a much stronger condition. (It is not difficult to see that an increasing union of open subsets of a complex space $X$, each one of them satisfying the continuous disk property, satisfies the continuous disk property. The proof is pretty much the same as the proof of Theorem 3.1, page 60, in [7.)

The following theorem was proved in [2] and [8].

Theorem 2.3. Let $\pi: X \rightarrow T$ be a proper holomorphic surjective map of complex spaces, let $t_{0} \in T$ be any point, and denote by $X_{t_{0}}:=\pi^{-1}\left(t_{0}\right)$ the fiber of $\pi$ at $t_{0}$. Assume that $\operatorname{dim} X_{t_{0}}=1$. Let $\sigma: \tilde{X} \rightarrow X$ be a covering space and let $\tilde{X}_{t_{0}}=$ $\sigma^{-1}\left(X_{t_{0}}\right)$. If $\tilde{X}_{t_{0}}$ is holomorphically convex, then there exists an open neighborhood $\Omega$ of $t_{0}$ such that $(\pi \circ \sigma)^{-1}(\Omega)$ is holomorphically convex.

The next result was proved in [1].

Proposition 2.4. Let $X$ be a 1-convex manifold with the exceptional set $S$ and let $p: \tilde{X} \rightarrow X$ be any covering. Then there exists a strongly plurisubharmonic function 
$\tilde{\phi}: \tilde{X} \rightarrow[-\infty, \infty)$ such that $p^{-1}(S)=\{\tilde{\phi}=-\infty\}$, and for any open neighborhood $U$ of $S$, the restriction $\tilde{\phi}_{\mid \tilde{X} \backslash p^{-1}(U)}$ is an exhaustion function on $\tilde{X} \backslash p^{-1}(U)$.

We recall that a topological space $X$ is called an ENR (Euclidean Neighborhood Retract) if it is homeomorphic to a closed subset $X_{1}$ of $\mathbb{R}^{n}$ for some $n$ such that there is an open neighborhood $V$ of $X_{1}$ and a continuous retract $\rho: V \rightarrow X_{1}$. For basic results regarding ENR's we refer to [5]. It is proved there that a locally compact and locally contractible subset of $\mathbb{R}^{n}$ is and ENR and that if a Hausdorff topological space $X$ is covered by countable family of locally compact open subsets each one of them homeomorphic with a subset of fixed Euclidean space, then $X$ is an ENR. In particular, every complex space of bounded dimension is an ENR.

Lemma 2.5 was proved in 3 .

Lemma 2.5. If $X$ is an ENR and $\left\{\gamma_{n}\right\}_{n \geq 1}, \gamma_{n}: S^{1}: \rightarrow X$, is a sequence of nullhomotopic loops converging uniformly to $\gamma: S^{1} \rightarrow X$, then $\gamma$ is null-homotopic. Moreover, given a covering $p: \tilde{X} \rightarrow X$ there exist liftings $\tilde{\gamma}_{n}$ for $\gamma_{n}$ and $\tilde{\gamma}$ for $\gamma$ such that $\tilde{\gamma}_{n}$ converges uniformly to $\tilde{\gamma}$.

This lemma can be applied in particular if $X$ is a complex space (by the above discussion).

Definition 2.6. Let $L$ be a connected 1-dimensional complex space and $\bigcup L_{i}$ be its decomposition into irreducible components. $L$ is called an infinite Nori string if all $L_{i}$ are compact and $L$ is not compact

It is clear that a 1-dimensional complex space is holomorphically convex if and only if it does not contain an infinite Nori string.

We recall that a compact complex curve is called rational if its normalization is $\mathbb{P}^{1}$

\section{The RESUlts}

Lemma 3.1. If $L$ is a 1-dimensional complex space that does not contain an infinite Nori string of rational curves, then $L$ has a holomorphically convex covering space.

Proof. Step 1. We assume that $L$ is connected and all its irreducible components are compact not rational. Let $L=\bigcup_{i \in I} L_{i}$ be the decomposition of $L$ into irreducible components and $A=\left\{x \in L: \exists i_{1}, i_{2} \in I, i_{1} \neq i_{2}\right.$, such that $\left.x \in L_{i_{1}} \cap L_{i_{2}}\right\}$. Let $p_{i}: \tilde{L}_{i} \rightarrow L_{i}$ be the universal cover of $L_{i}$. Since $L_{i}$ is not rational for all $i \in I$ we have that $\tilde{L}_{i}$ is Stein. For each $x \in A \cap L_{i}$, we choose a bijection $\tau(x, i): \mathbb{Z} \rightarrow p_{i}^{-1}(x)$ and we write $\tau(x, i)(k)=(k, x, i)$.

On the disjoint union $S:=\bigsqcup_{i \in I} \tilde{L}_{i}$ we define the projection $\tilde{p}: S \rightarrow L$ induced by $p_{i}, i \in I$. We also define an equivalence relation on $S$ by defining the equivalence classes as follows: if $s \in S$ is such that $\tilde{p}(s) \notin A$, then $\hat{s}=\{s\}$. Note that on $\tilde{p}^{-1}(A)$ we have a map $\zeta: \tilde{p}^{-1}(A) \rightarrow \mathbb{Z}$ which is nothing other than the projection on the first component. Then if $\tilde{p}(s) \in A$ and $\zeta(s)=k$ we set $\hat{s}=\left\{(k, x, i): x \in L_{i}, i \in\right.$ $I, \tilde{p}(s)=x\}$. We let $\tilde{L}$ be the quotient space of $S$ and $p: \tilde{L} \rightarrow L$ the application induced by $\tilde{p}$. It is not difficult to see that $p$ is a covering and $\tilde{L}$ is Stein.

Step 2. All irreducible components of $L$ are compact and not rational. In this case we apply the above procedure to each connected component.

Step 3. The general case. Let $L^{\prime}$ be the union of all irreducible components of $L$ that are compact and not rational and let $\left\{C_{j}\right\}_{j \in J}$ be the connected components 
of $L^{\prime}$. For each $j \in J$ let $p_{j}: \tilde{C}_{j} \rightarrow C_{j}$ be the Stein coverings obtained at the first step. Also, let $L^{\prime \prime}$ be the union of the other irreducible components of $L$. We set $\Gamma_{j}=L^{\prime \prime} \cap C_{j}$. (Note that $\Gamma_{j}$ might be empty.) If $\Gamma_{j} \neq \emptyset$ we consider in $\tilde{C}_{j}$ infinitely many disjoint copies $\left\{\Gamma_{j}^{\alpha}: \alpha \in \mathbb{Z}\right\}$ of $\Gamma_{j}$. (Each $\Gamma_{j}^{\alpha}$ is in bijection via $p_{j}$ with $\Gamma_{j}$.) We now glue infinitely many copies $\left\{L_{\alpha}^{\prime \prime}: \alpha \in \mathbb{Z}\right\}$ of $L^{\prime \prime}$ to $\tilde{C}_{j}$ on $\left\{\Gamma_{j}^{\alpha}: \alpha \in \mathbb{Z}\right\}$, and we obtain in this way a holomorphically convex covering of $L$.

Lemma 3.2. Suppose that $\tilde{X}$ and $X$ are Hausdorff topological spaces and $p: \tilde{X} \rightarrow$ $X$ is a covering. Let $\tilde{\gamma}_{n}: S^{1} \rightarrow \tilde{X}, \gamma_{n}: S^{1} \rightarrow X, n \geq 1, \tilde{\gamma}: S^{1} \rightarrow \tilde{X}, \gamma: S^{1} \rightarrow X$ be continuous functions such that $\gamma_{n}=p \circ \tilde{\gamma}_{n}, \gamma=p \circ \tilde{\gamma}$ and $a \in S^{1}$ be a fixed point. If $\gamma_{n}$ converges uniformly to $\gamma$ and $\tilde{\gamma}_{n}(a)$ converges to $\tilde{\gamma}(a)$, then $\tilde{\gamma}_{n}$ converges uniformly to $\tilde{\gamma}$.

Proof. Let $\Omega=\left\{t \in S^{1}: \lim _{n \rightarrow \infty} \tilde{\gamma}_{n}(t)=\tilde{\gamma}(t)\right\}$. We will show first that $\Omega=S^{1}$. As $a \in \Omega$ we have that $\Omega \neq \emptyset$. We prove that $\Omega$ is open in $S^{1}$. Let $t_{0} \in \Omega$ and let $V_{0}$ a neighborhood of $\gamma\left(t_{0}\right)$ which is evenly covered by $\bigcup \tilde{V}_{k}$. Let $\Omega_{0}$ be a connected open neighborhood of $t_{0}$ such that for $n$ large enough $\gamma_{n}\left(\Omega_{0}\right) \subset V_{0}$ and $\gamma\left(\Omega_{0}\right) \subset V_{0}$. It follows that $\tilde{\gamma}_{n}\left(\Omega_{0}\right) \subset \bigcup \tilde{V}_{k}$. As $\tilde{\gamma}_{n}\left(\Omega_{0}\right)$ and $\tilde{\gamma}\left(\Omega_{0}\right)$ are connected we have that for each $n$ there exists $k_{n}$ such that $\tilde{\gamma}_{n}\left(\Omega_{0}\right) \subset \tilde{V}_{k_{n}}$ and there exists $k_{0}$ such that $\tilde{\gamma}\left(\Omega_{0}\right) \subset \tilde{V}_{k_{0}}$. However, we assumed that $t_{0} \in \Omega$, and therefore for $n$ large enough $\tilde{\gamma}_{n}\left(t_{0}\right) \in \tilde{V}_{k_{0}}$, hence $V_{k_{n}}=V_{k_{0}}$. Since $p: V_{k_{0}} \rightarrow V_{0}$ is a homeomorphism we conclude that $\tilde{\gamma}_{n}$ converges uniformly to $\tilde{\gamma}$ on $\Omega_{0}$. This shows that $\Omega_{0} \subset \Omega$, and hence $\Omega$ is open.

Next we show in a similar fashion that $\Omega$ is closed. Let $t_{0} \in S^{1} \backslash \Omega$. As before we choose $V_{1}$ to be a neighborhood of $\gamma\left(t_{0}\right)$ which is evenly covered by $\bigcup \tilde{V}_{k}, \Omega_{0}$ a connected open neighborhood of $t_{0}$ such that for $n$ large enough $\gamma_{n}\left(\Omega_{0}\right) \subset V_{0}$, $k_{n}$ and $k_{0}$ such that $\tilde{\gamma}_{n}\left(\Omega_{0}\right) \subset \tilde{V}_{k_{n}}$ and $\tilde{\gamma}\left(\Omega_{0}\right) \subset \tilde{V}_{k_{0}}$. If $V_{k_{n}}=V_{k_{0}}$ for every $n$ we would have that $\tilde{\gamma}_{n}$ converges uniformly to $\tilde{\gamma}$ on $\Omega_{0}$ and hence at $t_{0}$, and this would contradict $t_{0} \notin \Omega$. There then exists a subsequence $\tilde{\gamma}_{n_{p}}$ such that $\tilde{\gamma}_{n_{p}}\left(\Omega_{0}\right) \cap \tilde{V}_{k_{0}}=\emptyset$, and from here we get that $\Omega_{0} \subset S^{1} \backslash \Omega$. Hence $\Omega$ is closed, and therefore $\Omega=S^{1}$.

Note that when we proved that $\Omega$ is open we actually proved that each $t_{0} \in$ $\Omega=S^{1}$ has an open neighborhood $\Omega_{0}$ such that on this neighborhood $\tilde{\gamma}_{n}$ converges uniformly to $\tilde{\gamma}$. The compactness of $S^{1}$ then implies that $\tilde{\gamma}_{n}$ converges uniformly to $\tilde{\gamma}$ on $S^{1}$.

Lemma 3.3. Suppose that $S$ is a complex space that has a holomorphically convex covering $p: \tilde{S} \rightarrow S$. If $\Omega \subset \mathbb{C}$ is an open neighborhood of $\bar{\Delta}$ and $f_{n}: \Omega \rightarrow S$ is a sequence of holomorphic functions which converges uniformly on $\{z \in \mathbb{C}:|z|=1\}$, then $\bigcup_{n} f_{n}(\bar{\Delta})$ is relatively compact in $S$. (In particular, $S$ satisfies the discrete disk property.)

Proof. Let $\gamma:\{z \in \mathbb{C}:|z|=1\} \rightarrow S$ be the limit of $\left\{f_{n}\right\}$ on $\{z \in \mathbb{C}:|z|=1\}$. It follows from Lemma 2.5 that $\gamma$ is null-homotopic. We choose a point $a \in\{z \in \mathbb{C}$ : $|z|=1\}$. Let $\epsilon$ be a positive number such that $\Delta_{1+\epsilon} \subset \Omega, \tilde{f}_{n}: \Delta_{1+\epsilon} \rightarrow \tilde{S}$ and let $\tilde{\gamma}:\{z \in \mathbb{C}:|z|=1\} \rightarrow \tilde{S}$ be liftings of $f_{n}$ and $\gamma$ respectively. We choose $\tilde{f}_{n}$ and $\tilde{\gamma}$ such that $\tilde{f}_{n}$ converges uniformly to $\tilde{\gamma}$ on $\{z \in \mathbb{C}:|z|=1\}$. This is possible by Lemma 2.5. As $\tilde{S}$ is holomorphically convex this implies that $\bigcup_{n} \tilde{f}_{n}(\bar{\Delta})$ is relatively compact in $\tilde{S}$ and therefore $\bigcup_{n} f_{n}(\bar{\Delta})$ is relatively compact in $S$. 
Theorem 3.4. Let $X$ be a 1-convex surface and $p: \tilde{X} \rightarrow X$ be a covering map. If $\tilde{X}$ does not contain an infinite Nori string of rational curves, then $\tilde{X}$ satisfies the discrete disk property.

Proof. Let $L$ be the exceptional set of $X$. Without loss of generality we may assume that $L$ is connected. Let $Y$ be a normal Stein complex space of dimension 2 and let $\pi: X \rightarrow Y$ be a proper surjective holomorphic map such that $\pi(L)=\left\{y_{0}\right\}$ and $\pi: X \backslash L \rightarrow Y \backslash\left\{y_{0}\right\}$ is a biholomorphism. We may assume that $Y$ is an analytic closed subset of $\mathbb{C}^{N}$.

We put $\tilde{L}:=p^{-1}(L)$. Since we assumed that $\tilde{L}$ does not contain an infinite Nori string of rational curves, it follows from Lemma 3.1 that $\tilde{L}$ has a holomorphically convex covering $\hat{p}: \hat{L} \rightarrow \tilde{L}$. We choose $U_{1}$ an open neighborhood of $L$ in $X$ that has a continuous deformation retract on $L$. We let $\tilde{U}_{1}=p^{-1}\left(U_{1}\right)$, and then $\tilde{U}_{1}$ has a continuous deformation retract on $\tilde{L}, \rho: \tilde{U}_{1} \rightarrow \tilde{L}$. By considering the fiber product of $\rho$ and $\hat{p}$ we obtain a covering $\hat{U}_{1}$ of $\tilde{U}$ such that the pull-back of $\tilde{L}$ is $\hat{L}$. We then apply Theorem 2.3 and we deduce that there exists $U \subset Y$ an open neighborhood of $y_{0}$ such that $(\pi \circ p)^{-1}(U)$ has a holomorphically convex covering.

Let $f_{n}: \Delta_{1+\epsilon} \rightarrow \tilde{X}, n \geq 1$, be a sequence of holomorphic functions such that $\bigcup_{n \geq 1} f_{n}\left(\Delta_{1+\epsilon} \backslash \Delta\right)$ is relatively compact in $\tilde{X}$ and $f_{n \mid S^{1}}$ is uniformly convergent. We argue by contradiction, and we assume that $\bigcup_{n \geq 1} f_{n}(\bar{\Delta})$ is not relatively compact in $\tilde{X}$ and hence that $\bigcup_{n \geq 1} f_{n}\left(\Delta_{1+\epsilon}\right)$ is not relatively compact. By passing to a subsequence we may assume that $\cup_{k \geq 1} f_{n_{k}}\left(\Delta_{1+\epsilon}\right)$ is not relatively compact in $\tilde{X}$ for every subsequence $\left\{f_{n_{k}}\right\}_{k}$ of $\left\{f_{n}\right\}_{n}$.

Since $\bigcup\left(\pi \circ p \circ f_{n}\right)\left(\Delta_{1+\epsilon} \backslash \Delta\right)$ is relatively compact in $Y$, and hence in $\mathbb{C}^{N}$, by the maximum modulus principle we have that $\bigcup\left(\pi \circ p \circ f_{n}\right)\left(\Delta_{1+\epsilon}\right)$ is relatively compact in $Y$. Then it follows that there exists a subsequence of $\left\{f_{n}\right\}_{n},\left\{f_{n_{k}}\right\}_{k}$ such that $\pi \circ p \circ f_{n_{k}}$ converges uniformly on compacts to a holomorphic function $g: D_{\epsilon} \rightarrow Y$. Without loss of generality we can assume that $\pi \circ p \circ f_{n}$ converges uniformly on compacts to $g$. We distinguish three cases.

Case 1. $g \equiv y_{0}$. Then $\left(\pi \circ p \circ f_{n}\right)\left(\bar{\Delta}_{1+\epsilon_{1}}\right) \subset U$ for $\epsilon_{1} \in(0, \epsilon)$ and $n$ large enough. Therefore we can apply Lemma 3.3

Case 2. $g(z) \neq y_{0}$ for every $z \in \Delta_{1+\epsilon}$. Then there exists a neighborhood $V$ of $y_{0}$ and $\epsilon_{1} \in(0, \epsilon)$ such that $g\left(\bar{\Delta}_{1+\epsilon_{1}}\right) \cap \bar{V}=\emptyset$. Then for $n$ large enough we get that $\pi \circ p \circ f_{n}\left(\bar{\Delta}_{1+\epsilon_{1}}\right) \cap \bar{V}=\emptyset$ and hence $f_{n}\left(\bar{\Delta}_{1+\epsilon_{1}}\right) \cap(\pi \circ p)^{-1}(V)=\emptyset$. We consider now a plurisubharmonic function $\tilde{\phi}$ on $\tilde{X}$ with the properties given in Proposition 2.4 In particular, its restriction to $\tilde{X} \backslash(\pi \circ p)^{-1}(V)$ is an exhaustion. Applying the maximum principle to $\tilde{\phi} \circ f_{n}$ we obtain immediately that $\bigcup f_{n}(\bar{\Delta})$ is relatively compact.

Case 3. $g \not \equiv y_{0}$ and $y_{0} \in g\left(\Delta_{1+\epsilon}\right)$. Then $g^{-1}\left(y_{0}\right)$ is a (non-empty) discrete subset of $\Delta_{1+\epsilon}$, and therefore there exists $\epsilon_{1} \in(0, \epsilon)$ such that $g^{-1}\left(y_{0}\right) \cap\{z \in \mathbb{C}:|z|=$ $\left.1+\epsilon_{1}\right\}=\emptyset$. We set $g^{-1}\left(y_{0}\right) \cap\left\{z \in \mathbb{C}:|z|<1+\epsilon_{1}\right\}=\left\{\lambda_{1}, \lambda_{2}, \ldots, \lambda_{s}\right\}$. Let $r_{1}, \ldots, r_{s}$ be positive numbers such that the discs $\bar{\Delta}_{j}=\left\{z \in \mathbb{C}:\left|z-\lambda_{j}\right| \leq r_{j}\right\}$, $j=1,2, \ldots, s$, are pairwise disjoint, $\bar{\Delta}_{j} \subset \Delta_{1+\epsilon_{1}}$ and $g\left(\bar{\Delta}_{j}\right) \subset U$. Let $V \subset Y$ be an open neighborhood of $y_{0}$ such that $\bar{V} \subset U$ and

$$
g\left(\left\{z \in \bar{\Delta}_{1+\epsilon_{1}}:\left|z-\lambda_{j}\right| \geq r_{j} \forall j=1, \ldots, s\right\}\right) \cap \bar{V}=\emptyset .
$$


Then for $n$ large enough we have that

$$
\left(\pi \circ p \circ f_{n}\right)\left(\left\{z \in \bar{\Delta}_{1+\epsilon_{1}}:\left|z-\lambda_{j}\right| \geq r_{j} \forall j=1, . ., s\right\}\right) \cap \bar{V}=\emptyset .
$$

We again apply Proposition 2.4 and we obtain a plurisubharmonic function $\tilde{\phi}$ : $\tilde{X} \rightarrow[-\infty, \infty)$ such that $\tilde{L}=\{\tilde{\phi}=-\infty\} \tilde{\phi}_{\mid \tilde{X} \backslash(\pi \circ p)^{-1}(V)}$ is an exhaustion function on $\tilde{X} \backslash(\pi \circ p)^{-1}(V)$. Since $\bigcup f_{n}\left(\Delta_{1+\epsilon} \backslash \Delta\right)$ is relatively compact there exists a positive constant $M$ such that $\tilde{\phi} \circ f_{n}(z) \leq M$ for every $n$ and every $z \in \Delta_{1+\epsilon} \backslash \Delta$. From the plurisubharmonicity of $\tilde{\phi}$ we have that $\tilde{\phi} \circ f_{n}(z) \leq M$ for every $z \in \Delta_{1+\epsilon}$, hence in particular for $z \in \bar{\Delta}_{1+\epsilon_{1}} \backslash \bigcup_{j=1}^{s} \Delta_{j}$. As $f_{n}\left(\bar{\Delta}_{1+\epsilon_{1}} \backslash \bigcup_{j=1}^{s} \Delta_{j}\right) \subset \tilde{X} \backslash(\pi \circ p)^{-1}(V)$ and $\tilde{\phi}_{\mid \tilde{X} \backslash(\pi \circ p)^{-1}(V)}$ is an exhaustion, we deduce that $\bigcup f_{n}\left(\bar{\Delta}_{1+\epsilon_{1}} \backslash \bigcup_{j=1}^{s} \Delta_{j}\right)$ is relatively compact in $\tilde{X}$.

For $j=1, \ldots, s$ we set $S_{j}=\left\{z \in \Delta_{1+\epsilon_{1}}:\left|z-\lambda_{j}\right|=r_{j}\right\}$ and we pick a point $a_{j} \in S_{j}$. As $\bigcup_{n>1} f_{n}\left(S_{j}\right)$ is relatively compact, by passing to a subsequence we can assume that each sequence $\left\{f_{n}\left(a_{j}\right)\right\}_{n}$ is convergent and we denote by $x_{j}$ its limit. We have that $\left(\pi \circ p \circ f_{n}\right)_{\mid S_{j}}$ converges uniformly to $g_{\mid S_{j}}$ and $\left(\pi \circ p \circ f_{n}\right)\left(S_{j}\right) \subset Y \backslash\left\{y_{0}\right\}$, $g\left(S_{j}\right) \subset Y \backslash\left\{y_{0}\right\}$. Because $\pi: X \backslash L \rightarrow Y \backslash\left\{y_{0}\right\}$ is a biholomorphism we deduce that $\left(p \circ f_{n}\right)_{\mid S_{j}}$ converges uniformly to $\left(\pi^{-1} \circ g\right)_{\mid S_{j}}$. Note now that $\left(p \circ f_{n}\right)_{\mid S_{j}}$ is in fact a null-homotopic loop. It follows from Lemma 2.5 that $\left(\pi^{-1} \circ g\right)_{\mid S_{j}}$ is null-homotopic as well, and therefore there exists a loop $\gamma_{j}: S_{j} \rightarrow \tilde{X}$ such that $p \circ \gamma_{j}=\left(\pi^{-1} \circ g\right)$ on $S_{j}$. From Lemma 3.2 we conclude that $f_{n}$ converges uniformly to $\gamma_{j}$ on $S_{j}$. Finally, Lemma 3.3 implies that $\bigcup f_{n}\left(\bar{\Delta}_{j}\right)$ is relatively compact for every $j=1, \ldots, s$.

\section{REFERENCES}

1. M. Colţoiu: Coverings of 1-convex manifolds with 1-dimensional exceptional set. Comment. Math. Helv. 68 (1993), no. 3, 469-479. MR.1236764 (95g:32023)

2. M. Colţoiu; K. Diederich: On the coverings of proper families of 1-dimensional complex spaces. Michigan Math. J. 47 (2000), no. 2, 369-375. MR.1793631 (2002f:32022)

3. M. Colţoiu; M. Tibăr: On the disk theorem. Math. Ann. 345 (2009), no. 1, 175-183. MR 2520056

4. F. Docquier; H. Grauert: Levisches Problem und Rungescher Satz für Teilgebiete Steinscher Mannigfaltigkeiten. Math. Ann. 140 (1960) 94-123. MR0148939 (26:6435)

5. A. Dold: Lectures on algebraic topology. Springer-Verlag, Berlin, 1995. MR 1335915 (96c:55001)

6. J. E. Fornaess: 2-dimensional counterexamples to generalizations of the Levi problem. Math. Ann. 230 (1977), no. 2, 169-173. MR0486625 (58:6344)

7. K. Fritzsche; H. Grauert: From holomorphic functions to complex manifolds. Graduate Texts in Mathematics, 213. Springer-Verlag, New York, 2002. MR1893803 (2003g:32001)

8. T. Napier: Convexity properties of coverings of smooth projective varieties. Math. Ann. 28 (1990), no. 1-3, 433-479. MR1032941 (91g:32015)

9. R. Narasimhan: The Levi problem for complex spaces. II. Math. Ann. 146 (1962) 195-216. MR0182747 (32:229)

"Simion Stollow" Institute of Mathematics of the Romanian Academy, P. O. Box 1-764, Bucharest 014700, Romania

E-mail address: Mihnea.Coltoiu@imar.ro

"Simion Stoilow" Institute of Mathematics of the Romanian Academy, P. O. Box 1-764, Bucharest 014700, Romania

E-mail address: Cezar.Joita@imar.ro 